\title{
La quemadura eléctrica por alto voltaje es un factor predictor de mortalidad en pacientes "grandes quemados"
}

\author{
GÜNTHER MANGELSDORFF G. ${ }^{1}$, \\ M. ANGÉLICA GARCÍA-HUIDOBRO D., ,a, ISAAC NACHARI B., 3, , \\ OMAR ATENAS M. ${ }^{4}$, SANDRA WHITTLE V. ${ }^{4}$, JORGE VILLEGAS C. ${ }^{4}$
}

\section{High voltage electrical burns as a risk factor for mortality among burn patients}

Background: Electrical burns are associated with complications and may aggravate burned patients. Aim: To evaluate the effect of electrical burns in mortality and length of stay in a critical care unit for burn patients. Material and Methods: Retrospective analysis of medical records of 182 patients aged 15 to 90 years, admitted to an intensive care unit for burn patients. The length of stay and mortality of $14 \mathrm{pa}$ tients that suffered electric burns was compared with the features of 168 patients with other type of burns. Results: Patients with electrical burns were younger, had a lower percentage of total body surface burnt and had a lower frequency of inhalatory injuries than their counterparts with other type of burns. Mortality rate among patients with electric or other types of burns was similar (three and 49 patients, respectively). Intensive care unit stay was also similar. A multivariate analysis showed that high voltage electric burns were an independent risk factor for death with an odds ratio of 12 (95\% confidence intervals 1.8-79.4). Conclusions: High voltage electric burns are an independent risk factor for death among burn patients.

(Rev Med Chile 2011; 139: 177-181).

Key words: Burns, electric; Intensive care; Wounds and injuries.

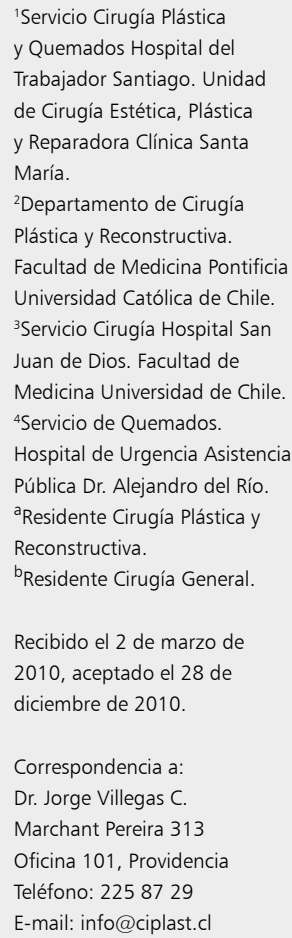

L a estimación del riesgo de muerte y la predicción de estadía en cuidados intensivos son elementos fundamentales para una planificación estratégica del tratamiento de pacientes "grandes quemados" (GQ). En años recientes ha sido posible generar modelos de predicción precisos y objetivos en pacientes GQ, lo que ha permitido orientar la estrategia de tratamiento, optimizar la asignación de los recursos y comprender la contribución relativa de criterios pronósticos específicos ${ }^{1-4}$.

Los factores clínicos predictores de mortalidad en quemados han sido establecidos en forma consistente en estudios previos ${ }^{1-9}$. Entre estos factores se encuentran variables como edad, sexo, superficie corporal quemada (SCQ), profundidad de las quemaduras y la presencia de injuria inhalatoria.
Sin embargo, existe poca información respecto al rol que podría tener la forma en que se produjo la quemadura en la predicción de resultados en GQ, especialmente en quemaduras eléctricas por alto voltaje. Estas quemaduras son consideradas tradicionalmente graves, ya que los pacientes sufren numerosos daños sistémicos y locales secundarios tanto al paso de la corriente eléctrica como a lesiones por arco voltaico y también por "fogonazo". Además, es frecuente la asociación de quemaduras eléctricas con otras lesiones traumáticas de alto riesgo de morbilidad y mortalidad (por ej: traumatismo encéfalo craneano, politraumatismo). De esta forma, en pacientes quemados por electricidad de alto voltaje, las alteraciones funcionales y de estructuras profundas serían mayores que el daño cutáneo ${ }^{10}$. A pesar de 
esto, la gravedad de los pacientes GQ es estimada actualmente según indicadores que no consideran la etiología como un factor predictor.

Nuestra hipótesis es que la quemadura eléctrica por alto voltaje es un factor predictor independiente de mayor mortalidad y morbilidad en estos pacientes. El objetivo de este estudio fue evaluar el efecto de la quemadura eléctrica por alto voltaje en la mortalidad intrahospitalaria y estadía en Unidad de Paciente Crítico (UPC) de pacientes GQ.

\section{Pacientes y Método}

\section{Diseño y pacientes}

Se realizó un estudio de cohorte retrospectivo ${ }^{11}$. Fueron incluidos todos los pacientes GQ adultos que ingresaron en forma consecutiva a la UPC del Servicio de Quemados del Hospital de Urgencia Asistencia Pública entre septiembre 2006 y julio 2008 (Centro de Referencia Nacional del Gran Quemado adulto). Los criterios de ingreso a esta unidad se resumen en la Tabla 1. La información clínica fue obtenida de una base de datos prospectiva diseñada y utilizada específicamente para los pacientes de esta unidad. Se registraron las variables edad, sexo, etiología, SCQ (\%), superficie de quemadura profunda (\%) e injuria inhalatoria (diagnóstico clínico según circunstancias del accidente, manifestaciones clínicas o mediante fibrobroncoscopia). Se registró la estadía en la UPC, que incluyó la hospitalización en cuidados intensivos e intermedios y la mortalidad. El tiempo de seguimiento corresponde al período de estadía en la UPC. La muestra se dividió en dos grupos

Tabla 1. Criterios de ingreso a la Unidad de Paciente Crítico (UPC) del Servicio de Quemados del Centro de Referencia Nacional (Hospital de Urgencia Asistencia Pública)

\section{Criterios de ingreso a UPC}

$\mathrm{SCQ}^{\mathrm{a}}>20 \%$ de quemaduras intermedias o profundas Indice de gravedad $>70^{\mathrm{b}}$

Quemaduras respiratorias/inhalación de humo

Quemaduras eléctricas por alta tensión

Quemados politraumatizados

Quemados con patologías graves asociadas

a Superficie corporal quemada. ${ }^{b}$ Se utiliza el índice de gravedad de Garcés (2). según la etiología de la quemadura: 1) Grupo Quemaduras Eléctricas: GQ por quemadura eléctrica de alto voltaje; 2) Grupo Otras Causas: GQ con quemadura secundarias a fuego y escaldaduras. Se compararon las características clínicas, mortalidad y estadía en UPC entre ambos grupos. Se evaluó el efecto independiente del factor quemadura eléctrica como predictor de mortalidad y estadía en UPC.

\section{Protocolo de tratamiento}

Todos los pacientes fueron tratados según el protocolo establecido en la Guía Clínica "Gran Quemado" del Ministerio de Salud, con reanimación y soporte médico intensivo multidisciplinario, tratamiento quirúrgico basado en estrategia de protección de queratinocitos viables en quemaduras de espesor parcial (coberturas sintéticas semiporosas no adherentes), y en las quemaduras de espesor total escarectomía precoz asociada a cobertura transitoria (heteroinjertos/homoinjertos irradiados criopreservados) o definitiva (autoinjertos) $^{12}$.

Todos los pacientes o sus familiares autorizaron las cirugías y firmaron un consentimiento informado diseñado para este fin.

\section{Análisis estadístico}

Las variables categóricas se describieron con frecuencias (\%) y en la comparación de proporciones se utilizó el test exacto de Fisher. Dado que las variables continuas no presentaron distribución normal (Shapiro Wilk), fueron descritas con mediana y rangos (mínimo-máximo) y se compararon con test no paramétrico (U Mann Whitney). Se realizó al análisis multivariado del efecto de la variable quemadura eléctrica (presente/ausente) como predictor de mortalidad mediante regresión logística, y como predictor de estadía en UPC mediante regresión lineal múltiple. El análisis multivariado de sobrevida en la UPC se hizo mediante regresión de $\mathrm{Cox}^{13}$. Se expresan los valores de odds ratio e intervalos de confianza de 95\% ( OR $\left.\left[\mathrm{IC}_{95}\right]\right)$. Todos los modelos de regresión fueron ajustados según las covariables edad, sexo, SCQ (\%), superficie de quemadura profunda (\%) e injuria inhalatoria (presente/ausente). Se utilizaron estas covariables porque han sido previamente identificadas, en distintos estudios, como factores predictores de mortalidad. Se consideró significativo un valor $\mathrm{p}<0,05$ para dos colas. Se utilizó el programa SPSS 16.0.2 ${ }^{\circledR}$. 


\section{Resultados}

La muestra estuvo constituida por 182 pacientes, 57 mujeres (31\%) y 125 hombres $(69 \%)$, con una mediana de edad de 45 años (15-90). En 14 pacientes $(7,7 \%)$ la etiología fue quemadura eléctrica por alto voltaje (Grupo Quemaduras Eléctricas), mientras que en 168 (92,3\%) fue por otras causas (Grupo Otras Causas), entre los cuales se registraron 161 quemaduras por fuego (14 fueron autoinferidas) y 7 escaldaduras. En la Tabla 2 se puede apreciar que los pacientes del Grupo Quemaduras Eléctricas fueron significativamente menores, mayoritariamente hombres, tenían menor SCQ y menor frecuencia de injuria inhalatoria, que los pacientes del Grupo Otras Causas. No se observó diferencia en la superficie de quemadura profunda entre ambos grupos.

\section{Mortalidad y estadía en UPC}

La mortalidad global de la serie fue de 52 pacientes $(28,6 \%)$. En el análisis univariado no hubo diferencia en la mortalidad entre ambos grupos: quemaduras eléctricas $3 / 14(21 \%)$ vs otras causas 49/168 (29\%), $\mathrm{p}=0,760$.

La estadía en UPC global fue de 14 (1-136) días, sin diferencias entre ambos grupos: quemaduras eléctricas 8 (3-56) días vs otras causas 15 (1-136) días, $\mathrm{p}=0,212$.

\section{Riesgo de muerte y estadía en UPC según etiología de la quemadura}

En el análisis multivariado, ajustado por las covariables previamente detalladas en los métodos, se observó que el factor quemaduras eléctricas se asocia a mayor mortalidad intrahospitalaria (OR $11,978 \mathrm{IC}_{95 \%}[1,806-79,425], \mathrm{p}=0,010$, regresión logística). Sin embargo, este factor no se asoció al tiempo de estadía en UPC (OR -0,009 IC $_{95 \%}$ $[-16,825-15,072], \mathrm{p}=0,914)$.

\section{Sobrevida en la UPC según etiología de la quemadura}

Al analizar la sobrevida en la UPC (regresión de Cox), se observó que el factor quemadura eléctrica se asocia en forma significativa a mayor mortalidad (OR 7,858 IC $_{95 \%}[1,992-30,992], p$ $=0,003)$. En la Figura 1 se muestran las curvas del modelo obtenido de sobrevida en la UPC, con un tiempo de seguimiento de 14 (1-136) días, que corresponde al tiempo de estadía en la UPC. Según este modelo, el grupo de pacientes

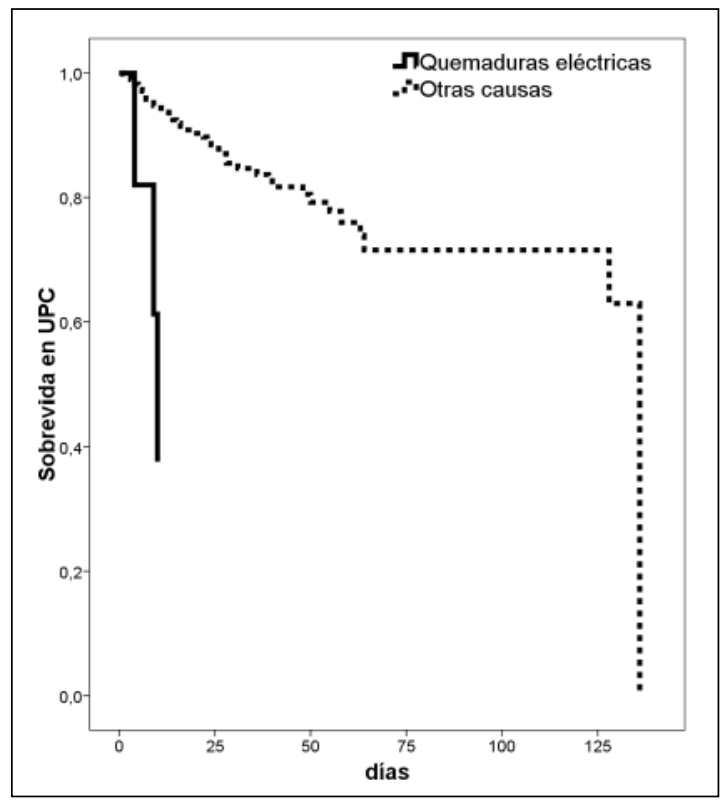

Figura 1. Curvas de sobrevida del modelo obtenido según regresión de Cox. Modelo ajustado por edad, sexo, SCQ (\%), SCQ profunda (\%) e injuria inhalatoria (presente/ausente). Se comparan las curvas de ambos grupos.

Tabla 2. Características clínicas de los pacientes de ambos grupos

\begin{tabular}{|lccc|}
\hline & $\begin{array}{c}\text { Quemaduras eléctricas } \\
\mathbf{n = 1 4}\end{array}$ & $\begin{array}{c}\text { Otras causas } \\
\mathbf{n = 1 6 8}\end{array}$ & Valor $\mathbf{p}$ \\
\hline Edad, años & $31(17-63)^{\mathrm{b}}$ & $45(15-90)^{\mathrm{b}}$ & 0.004 \\
Sexo, hombres/mujeres & $14 / 0$ & $111 / 57$ & 0.006 \\
SCQ ${ }^{\mathrm{b}}$ \% & $10(1-50)^{\mathrm{b}}$ & $20(1-95)^{\mathrm{b}}$ & 0.033 \\
SCQ profundaa $\%$ & $6(1-33)^{\mathrm{b}}$ & $1(0-95)^{\mathrm{b}}$ & 0.076 \\
Injuria inhalatoria, $\mathrm{n}(\%)$ & $1 / 14(7)$ & $68 / 168(40)$ & 0.019 \\
\hline
\end{tabular}

a Superficie Corporal Quemada. 'Los valores expresan la mediana y rango. 
con quemaduras eléctricas tiene una curva con un descenso más rápido. Por otra parte, la curva de sobrevida en UPC del grupo "otras causas" cae a cero en el seguimiento alejado, porque los únicos pacientes que registraron estadías en UPC tan prolongadas eran casos muy graves, que finalmente fallecieron.

\section{Discusión}

Este estudio muestra que la quemadura eléctrica por alto voltaje es un factor predictor de mortalidad en GQ adultos. Si bien las cifras de mortalidad observadas en ambos grupos fue similar, los pacientes que sufrieron quemaduras eléctricas eran hombres más jóvenes, con menos superficie quemada y con menos injuria inhalatoria que el grupo con otras etiologías. De esta forma, la mortalidad observada en el grupo de pacientes con quemaduras eléctricas por alto voltaje fue significativamente mayor a la esperada para su edad, sexo, superficie corporal quemada y proporción de injuria inhalatoria, lo que se traduce a un mayor riesgo ajustado de muerte. Esta asociación se confirmó tanto en análisis de regresión logística como en las diferencias de las curvas de sobrevida en la UPC.

Esta asociación no ha sido descrita previamente en estudios de análisis de predictores de mortalidad en pacientes quemados adultos, lo que se debe a la ausencia de estudios que hayan evaluado en forma dirigida el rol de la etiología en la mortalidad de pacientes quemados. La mayoría de los trabajos disponibles tienen importantes limitaciones en este sentido, ya sea porque no entregan detalles respecto a la etiología de las quemaduras revisadas, o porque la proporción de pacientes con quemaduras eléctricas es muy baja. Ryan y cols ${ }^{14}$ analizaron los factores predictores de mortalidad en 1.665 pacientes quemados admitidos al Instituto Shriners y al Hospital General de Massachussets entre 1990 y 1994. Los factores de riesgo identificados mediante regresión logística fueron edad $>60$ años, SCQ $>40 \%$ e injuria inhalatoria. El tipo de quemadura no se asoció en forma significativa a la mortalidad. En este estudio no fueron especificados los tipos de quemaduras registrados, así como tampoco la proporción de pacientes con quemaduras eléctricas que fueron incluidos. Además de presentar estas importan- tes limitaciones, estos resultados no se pueden comparar con los de nuestro trabajo, dado que las poblaciones son considerablemente diferentes. En la serie de Ryan y cols la muestra incluyó niños y adultos, de distintos niveles de gravedad, con una mortalidad global de 4\% (67/1665), lo que dificulta un buen análisis de predictores de muerte, dado que el evento estudiado fue de baja frecuencia en su serie ${ }^{15}$. Al contrario, en nuestro estudio sólo fueron incluidos pacientes adultos $\mathrm{GQ}$, quienes presentan una mayor gravedad y mortalidad ( $29 \%$ de mortalidad global), lo que permite un mejor estudio de factores predictores. Meshulam-Derazon y cols ${ }^{16}$ analizaron los predictores de mortalidad y morbilidad en 249 pacientes admitidos a la unidad de quemados de centro médico de Rabin (Telaviv) entre 1995 y 2002. La SCQ y la injuria inhalatoria fueron los únicos factores predictores independientes de mortalidad, y el tipo de quemadura no se asoció a mortalidad ni a morbilidad. En esta muestra, sólo $2 \%$ de las quemaduras fueron eléctricas. La falta de asociación entre el tipo de quemadura y la mortalidad/morbilidad que muestra este estudio no es concluyente, dado que se analizó un predictor representado en un bajo porcentaje de la muestra. En contraste con lo anterior, en nuestro estudio $7,7 \%$ de los pacientes presentaban quemaduras eléctricas por alto voltaje, lo que representa una proporción de casos que permite un mejor análisis del rol de la etiología en la mortalidad de estos pacientes.

Si bien presentamos una serie importante de pacientes GQ, derivados desde distintas partes de Chile al centro de referencia nacional, debemos destacar el bajo número de pacientes con quemaduras eléctricas incluidos en este estudio. Esto se debe a la baja frecuencia de este tipo de accidente. De todas formas, los hallazgos descritos son significativos, a pesar que el bajo número de casos reduce la precisión de los indicadores de riesgo obtenidos.

La estadía en cuidados intensivos es habitualmente utilizada como un indicador de gravedad de los pacientes. Esta relación se ve alterada en pacientes quemados, donde los casos más graves habitualmente fallecen en el período de tratamiento inicial. Para compensar esta limitación, algunos autores analizan la estadía hospitalaria excluyendo los casos de muertes precoces (primeras $48 \mathrm{hrs})^{14}$. En nuestro estudio no realizamos estas exclusiones para evitar sesgo en nuestra 
comparación, ya que los grupos de pacientes que analizamos podrían tener distintos perfiles temporales de mortalidad. De hecho, las curvas de sobrevida en la UPC muestran que las muertes en el grupo "quemaduras eléctricas" ocurrieron en forma más precoz. Considerando estas limitaciones, la variable estadía en UPC no refleja la gravedad de los pacientes incluidos en este estudio, pero entrega información relevante sobre la necesidad y uso de recursos sanitarios por parte de estos pacientes.

En nuestro estudio, la quemadura eléctrica por alto voltaje no se asoció a una mayor estadía hospitalaria, hallazgo que concuerda con lo publicado por otros autores ${ }^{7,16}$. Al ser un centro de referencia nacional, muchos de los pacientes que ingresan a nuestra unidad han iniciado su tratamiento en otro centro, donde a su vez regresan cuando se ha logrado la estabilización clínica y la cobertura cutánea completa. De esta forma, las conclusiones se ven limitadas en este punto, ya que una parte variable de la estadía en cuidados intensivos no ha sido incluida en este trabajo. Del mismo modo debemos tener presente esta limitación en el análisis de mortalidad ya que es posible que haya un subregistro de las muertes tardías, eventualmente ocurridas en los centros de destino.

Las quemaduras eléctricas de alto voltaje son consideradas graves y representan un criterio universalmente aceptado para definir el traslado de los pacientes a un centro especializado en quemados GQ. Nuestros hallazgos justifican plenamente esta conducta. La etiología de quemadura eléctrica debe ser considerada en forma paralela a los índices de gravedad de cada paciente, dado que ejerce un efecto independiente a las variables incluidas en los cálculos de estos índices (edad, sexo, SCQ, profundidad e injuria inhalatoria) $)^{1,2,4}$. Por esta razón planteamos que sería necesaria una actualización de los indicadores de gravedad, incluyendo la etiología como factor, y recomendamos considerar a los pacientes quemados eléctricos como un grupo de pacientes con peor pronóstico.

En suma, nuestro estudio muestra que la quemadura eléctrica por alto voltaje es un factor predictor de mayor mortalidad en GQ adultos. Esta información puede tener importantes aplicaciones, tanto en la toma de decisiones basada en estimación de gravedad, como en la búsqueda de elementos modificables que permitan mejorar el pronóstico de estos pacientes.

\section{Referencias}

1. Tobiasen J, Hiebert J, Edlich R. The abbreviated burn severity index. Ann Emerg Med 1982; 11: 260-2.

2. Garcés M. Pronóstico vital en quemados. En Garcés M, Artigas R, editores. Quemaduras. Santiago, Chile: Sociedad de Cirujanos de Chile; 1995. p. 79-83.

3. McGwin J, George R, Cross J, Rue L. Improving the ability to predict mortality among burn patients. Burns 2008; 34: 320-7.

4. Blot S, Brusselaers N, Monstrey S, Vandewoude K, De Waele J, Colpaert K, et al. Development and validation of a model for prediction of mortality in patients with acute burn injury. Br J Surg 2009; 96: 111-7.

5. Pruitt B, Tumbusch W, Mason A. Mortality in 1100 consecutive burns treated at a burns unit. Ann Surg 1964; 159: 396-401.

6. Zawacky B, Azen S, Imbus S, Chang Y. Multifactorial probit analysis of mortality in burned patients. Ann Surg 1979; 189: 1-5.

7. Curreri P, Literman A, Braun W. Burn injury. Analysis of survival and hospitalization time for 937 patients. Ann Surg 1980; 195: 472-8.

8. Lionelli G, Pickus E, Beckum O, Decoursey R, Korentager R. A three decade analysis of factors affecting burn mortality in the elderly. Burns 2005; 31: 958-63.

9. Mahar P, Wasiak J, Bailey M, Cleland H. Clinical factors affecting mortality in elderly burn patients admitted to a burns service. Burns 2008; 34: 629-36.

10. Koumbourlis C. Electrical Injuries. Crit Care Med 2002; 30: S424-30.

11. Cummings S, Newman T, Hulley S. Designing a Cohort Study. En: Hulley S, Cummings S, Browner W, Grady D, Newman T, editores. Designing Clinical Research. Philadelphia, USA: Lippincott Williams \& Wilkins; 2007. p. 97-108.

12. MINISTERIO DE SALUD. Guía Clínica Gran Quemado. Minsal, 2007

13. Friedman L, Furgberg C, DeMets D. Survival analysis. En: Friedman L, Furberg C, DeMets D, editors. Fundamentals of clinical trials, New York, USA: Springer, 1998. p. 223-45.

14. Ryan C, Schoenfeld D, Thorpe W, Sheridan R, Cassem E, Tompkins R. Objective estimates of the probability of death from burn injuries. N Engl J Med 1998; 338: 362-6.

15. Saffle J. Predicting outcomes of burns. N Engl J Med 1998; 338: 387-8.

16. Meshulam-Derazon S, Nachumovsky S, Ad-El D, Sulkes J, Hauben D. Prediction of morbidity an mortality on admission to a burn unit. Plast Reconstr Surg 2006; 118: 116-20. 